\title{
The Effect of Low Frequency Repetitive Transcranial Magnetic Stimulation Combined with Range of Motion Exercise on Paretic Hand Function in Female Patients after Stroke
}

\author{
Mee-Young Kim ${ }^{1}$, Ju-Hyun Kim ${ }^{1}$, Jeong-Uk Lee ${ }^{1}$, Byong-Yong Hwang ${ }^{2}$, Junghwan Kim ${ }^{2 *}$ \\ ${ }^{1}$ Laboratory of Health Science \& Nanophysiotherapy, Department of Physical Therapy, Graduate School, Yongin University, Yongin, \\ South Korea; ${ }^{2}$ Department of Physical Therapy, College of Public Health \& Welfare, Yongin University, Yongin, South Korea. \\ Email: junghwankim3@yongin.ac.kr
}

Received February $24^{\text {th }}, 2013$; revised March $25^{\text {th }}, 2013$; accepted April $15^{\text {th }}, 2013$

Copyright (C) 2013 Mee-Young Kim et al. This is an open access article distributed under the Creative Commons Attribution License, which permits unrestricted use, distribution, and reproduction in any medium, provided the original work is properly cited.

\begin{abstract}
Repetitive transcranial magnetic stimulation (rTMS) has recently been demonstrated to improve motor function after stroke. However, no study has yet tested the synergetic effects of physical exercise on rTMS in clinical settings. We investigated the effect of a 6-session course of low frequency rTMS on contralesional primary motor cortex combined with range-of motion (ROM) exercise on paretic hand function in female stroke patients. This was a single-blind study of the effects of rTMS with or without ROM exercise in female hemiplegic patients after stroke. All patients underwent rTMS on the contralesional primary motor cortex for 15 minutes and ROM exercise on the paretic hand. The cortical excitability determined by the amplitude and latency of the motor evoked potential (MEP) was measured in both first dorsal interosseous (FDI) muscles. We also evaluated arm function using Box and Block, arm reach, 9-hole pegboard, power grip, and pinch grip force tests. The rTMS-induced MEP amplitude of the paretic side significantly increased whereas the non-paretic side showed a decrease through every session. However, the MEP latency significantly increased on the non-paretic hand at post-rTMS with exercise, but a tendency of decrement on paretic hand at same application. Motor function showed improvement in the 9-hole pegboard and arm reach tests at post-rTMS with exercise on the paretic side compared with the non-paretic side. A significant correlation was especially noted between motor function and MEP on the paretic side of stroke patients. Low frequency rTMS with ROM exercise improved hand function after stroke. This may, in part, result in additional rehabilitation in stroke patients.
\end{abstract}

Keywords: Repetitive Transcranial Magnetic Stimulation; Range of Motion Exercise; Stroke; Motor Recover

\section{Introduction}

Patients after stroke commonly suffer from significant impairments including weakness, loss of voluntary motor control, and spasticity [1-3]. After completing standard rehabilitation, approximately $50 \%-60 \%$ of stroke patients still experience some degree of motor impairment [4], and at least $20 \%$ remain partially dependent in pursuing activities-of-daily-living (ADL) [5]. Physical therapy for stroke patients primarily concerns requisition of motor function to perform tasks and ADL such as grasping, reaching, and other more physical demanding movements [3]. A previous report has shown a developing role for physical therapy assistants [6], and some studies have

${ }^{*}$ Corresponding author. shown that physical therapy can increase upper extremity motor recovery $[7,8]$. Range of motion (ROM) exercise is commonly used in clinical settings and mainly aims to preserve flexibility and mobility of joints [9]. In particular, ROM exercise improves function, pain relief, ADL, and symptoms in stroke patients $[10,11]$. The interhemispheric competition model indicates that both hemispheres inhibit each other in a competition under natural conditions [12,13]. Brain injury results in inhibition of the primary motor cortex (M1) of the ipsilesional hemisphere by M1 of contralesional hemisphere without competition. This then causes an abnormal increase of transcallosal inhibition (TCI) from the contralesional hemisphere to the ipsilesional one [12,13]. Previous studies have reported that repetitive transcranial mag- 
netic stimulation (rTMS) of the contralesional M1 reduces TCI, thereby disinhibiting ipsilesional hemisphere, and lead to enhanced excitability of the ipsilesional hemisphere [12,13-15]. Treatment with rTMS can change excitability of the human cortex for at least several minutes and can influence the metabolic rate of the stimulated cortex area. Therefore, rTMS treatment may lead to functional change in the paretic hand $[15,16]$. We were interested in upper extremity motor function of chronic stroke patients after application of exercise and low frequency rTMS of the unaffected hemisphere to downregulate TCI. At present, TMS is not used directly with physical therapy [17], even though additional physical training can augment the effect of rTMS and it may contribute to functional recovery after stroke $[15,18]$. Therefore, as observed for rTMS, we hypothesized that changes in excitability of both hemispheres would be seen following ROM exercise on paretic upper extremity combined with rTMS on the unaffected M1. We examined whether ROM exercise combined with rTMS promotes functional improvement on paretic upper extremity and whether a change in cortical excitability is correlated with improvement of upper extremity reach and grasp in stroke patients.

\section{Materials and Methods}

We studied six female patients aged 45 to 75 years $(60.0$ \pm 5.0 years) at least 1 year after stroke (Table 1). Each had experienced a single ischemic stroke with more than 1 year of duration and each lesion site was located only in the subcortex, as confirmed by computed tomography (CT) or magnetic resonance imaging (MRI). Their motor deficits in the paretic upper extremity had recovered to the extent that patients could perform grasping tasks. The patients scored in the normal range of the Korean Mini-Mental State Examination. Every participant visited and underwent 6 treatment sessions. Each session included assessment of motor function using Box and Block, 9-hole pegboard, arm reach, power grip and pinch grip force tests. The cortical excitability was determined by motor evoked potential (MEP) amplitude and latency, which observed and rectified by electromyographic (EMG) signals of both first dorsal interosseous (FDI) muscles. Motor function was evaluated 2 times at preintervention and post-intervention 2 (post-rTMS + exercise) and cortical excitability was measured 3 times at pre-intervention, post-intervention 1 (post-rTMS), and post-rTMS + exercise in every single session. In total, motor function was evaluated 12 times, and MEP was measured 18 times ( 3 times in a session, in 6 sessions) in our experiment (Figure 1). A MAG PRO butterfly coil (MCF-B65) and MAG PRO R30 (Medtronic, Inc.) were used for determination of resting motor threshold (rMT)

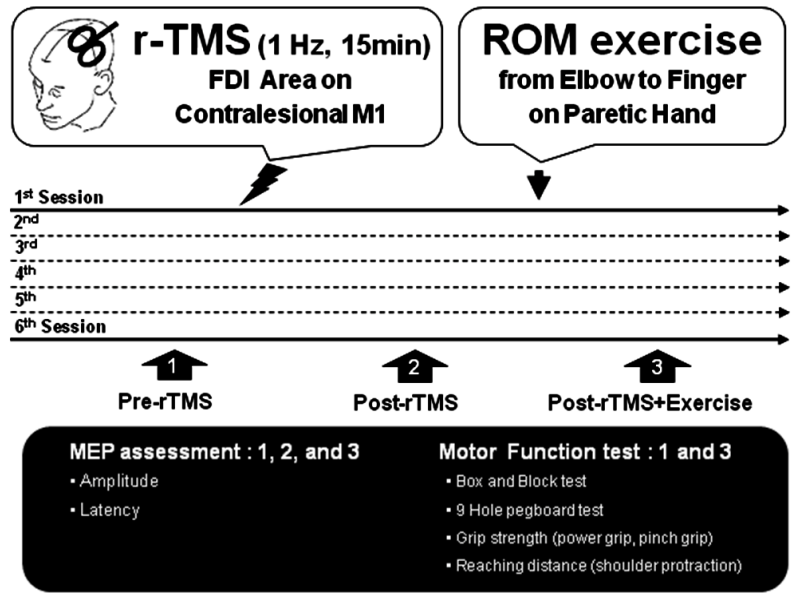

Figure 1. Schematic representation of the experimental procedure and time course. rTMS, repetitive transcranial magnetic stimulation; ROM exercise, range of motion exercise; FDI; first dorsal interosseous muscles; M1, primary motor cortex; MEP, motor evoked potential.

and conduction of rTMS for 15 minutes. The coil was placed tangentially over M1 at the optimal site for the FDI muscle during rTMS application. EMG activity was recorded from silver-silver-chloride electrodes positioned in a belly-tendon montage on skin overlying the FDI using KEYPOINT ${ }^{\circledR}$. NET software. The rMT was defined as the lowest stimulator output that could activate MEPs with a peak-to-peak amplitude $50 \mu \mathrm{V}$ in at least half of 10 trials. We applied rTMS $(1 \mathrm{~Hz}, 100 \%$ rMT, 15 minutes) on the contralesional M1. Immediately after the rTMS procedure, we conducted ROM exercise for 30 minutes on the paretic upper extremity (elbow flexion/ extension, forearm supination/pronation, wrist flexion/ extension, finger abduction/adduction, and thumb and index finger opposition) in the following order: active assistive exercise 10 times, active exercise 10 times, and resistive exercise 10 times on each movement (Figure 1). All subjects provided their informed consent to participation in the study, and the protocol for the study was approved by the Committee of Ethics in Research of the University of Yongin, in agreement with the terms of Resolution 5-1-20, December 2006. Data were expressed as mean \pm standard error (SE). The effect of ROM exercise combined with rTMS was evaluated with an ANOVA for repeated measures and one-way ANOVA. A post-hoc analysis was performed with a Scheffe $F$ test. Any possible correlation between changes in various parameters was determined using a Pearson correlation coefficient test.

\section{Results}

The rTMS, as measured in stroke patients, induced a decrease in MEP amplitude in the non-paretic hand, 
Table 1. Clinical characteristics of stroke patients.

\begin{tabular}{cccccc}
\hline Age $(\mathrm{yr})$ & Gender & Time post-stroke (mo) & K-MMSE (Score) & rMT (\%) & Lesion site \\
\hline $60.0 \pm 5.0$ & Female & $30.2 \pm 5.1$ & $28.5 \pm 0.8 / 30$ & $62.3 \pm 4.0$ & Middle cerebral artery Pons, Basal ganglia \\
\hline
\end{tabular}

K-MMSE, Korean version of mini mental status examination; rTM, Resting motor threshold.

whereas it led to an increment in MEP amplitude in the paretic hand at 3 time points (Figure 2). The occurring latency of MEP also was longer in the paretic hands of stroke patients compared with the non-paretic side (Figure 2). In the non-paretic side, the repetition of rTMS and exercise during the $6^{\text {th }}$ session elevated the time of latency. As shown in Figure 3(A), the representative results in the $3^{\text {rd }}$ session indicate that latency was increased by rTMS treatment. ROM exercise for $30 \mathrm{~min}$ after rTMS further increased the latency (Figures 2(A)

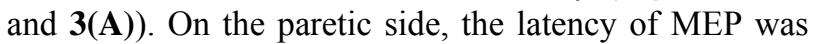
increased by the rTMS and ROM exercise, and was abolished by the repetition of rTMS and ROM exercise during the $6^{\text {th }}$ session (Figure 2(B)).

The amplitude of MEP was greater in the non-paretic hands of stroke patients compared with the paretic side (Figures 2, 3(B)-(a'), 3(B)-(b')). The rTMS and ROM exercise did not alter the amplitude of MEP in the $1^{\text {st }}$ session but repetition of the stimulation decreased it in the non-paretic side (Figure 3(B)). In particular, ROM exercise for $30 \mathrm{~min}$ after rTMS further decreased the amplitude of MEP in the non-paretic side (Figure 3(B)-(a')). In contrast, the amplitude of MEP was increased by the rTMS treatment, which was further elevated by the repetition of rTMS and ROM exercise during the $6^{\text {th }}$ session on the paretic side (Figure 3(B)-(b)). Through all 6 sessions, motor function was measured 12 times at pre-rTMS and at post-rTMS + exercise by Box and Block, 9-hole pegboard, arm reach, power grip force, and lateral pinch grip force tests (Table 2). Compared with the non-paretic side, the paretic side showed continuous motor function improvement in Box and Block and 9-hole pegboard tests through every session (Table 2). According to our results, overall performance time for the 9-hole pegboard test was significantly reduced especially for post-rTMS + exercise compared with pre-rTMS on the paretic hand (Table 2). The Box and Block test on the paretic side also showed a tendency toward overall improvement at post-rTMS + exercise compared with pre-rTMS on paretic side (Table 2). We analyzed the correlation between motor function and MEP parameters (Table 3). The 9-hole pegboard and arm reach test results were correlated with MEP amplitude, while the Box and Block, force of power grip, force of pinch grip, and arm reach tests were correlated with MEP latency on the paretic side. Only the pinch grip force was correlated with MEP amplitude on the non-paretic side (Table 3).
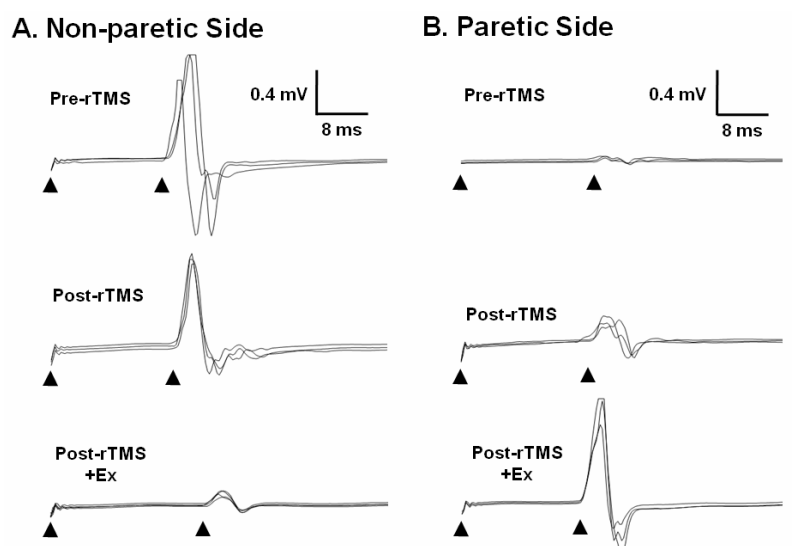

Figure 2. Diagram of MEP amplitude and latency from both first dorsal interosseous muscles. MEP amplitude and latency were determined, as described in the materials and methods. rTMS, repetitive transcranial magnetic stimulation; MEP, motor evoked potential.

\section{Discussion}

The present study provides the first direct demonstration that physical therapy combined with rTMS can facilitate cortical excitability and motor recovery in stroke patients. This study was based on the hypotheses that inhibition of TCI by rTMS would result in an increase in cortical excitability in the ipsilesional hemisphere and that ROM exercise added to rTMS would accelerate increases in cortical excitability that ultimately would translate into a motor improvement. We found a clear increase in MEP amplitude on the paretic side whereas the non-paretic hand showed a decrease. The degree of change after ROM exercise in the paretic hand greatly increased at the $6^{\text {th }}$ session. The changes in MEP amplitude between prerTMS and post-rTMS, on both FDIs, are in agreement with previous studies $[14,15,18]$. These results indicate that treatment with rTMS at $1 \mathrm{~Hz}$ over the contralesional hemisphere down-regulates TCI from the contralesional hemisphere to the ipsilesional hemisphere, which then leads to an increase in cortical excitability in the ipsilesional hemisphere of stroke patients. Interestingly, the MEP amplitude of paretic FDI at post-rTMS + exercise significantly increased and to a greater extent than was seen at post-rTMS, with the greatest increase seen at post-rTMS + exercise at the $6^{\text {th }}$ session. This suggests that physical therapy combined with rTMS may be more beneficial than rTMS alone and that the effect may be cumulative over long-term treatment. No previous study 
A. MEP-Latency

\section{a) Non-paretic}

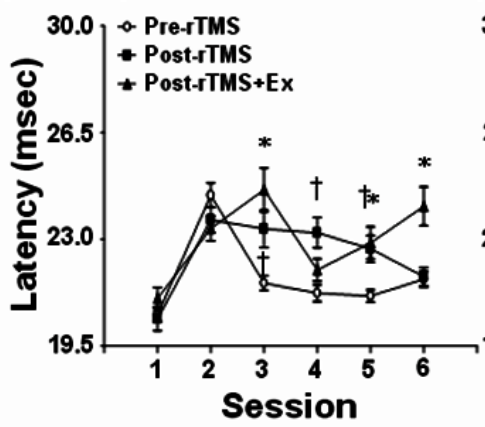

a' ) Total

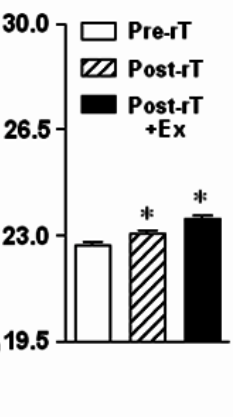

b) Paretic

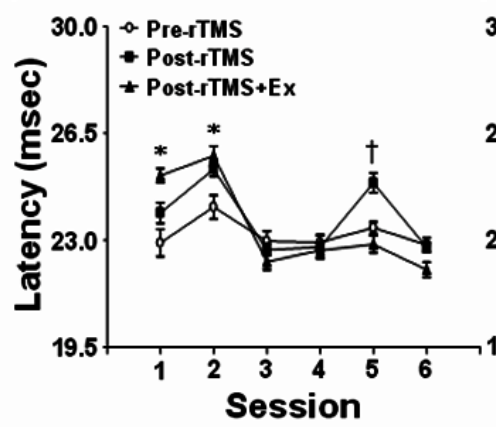

b') Total

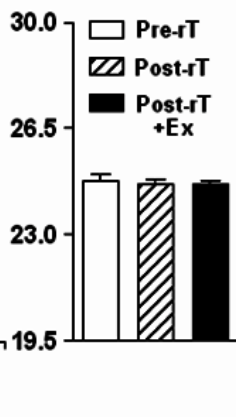

B. MEP-Amplitude

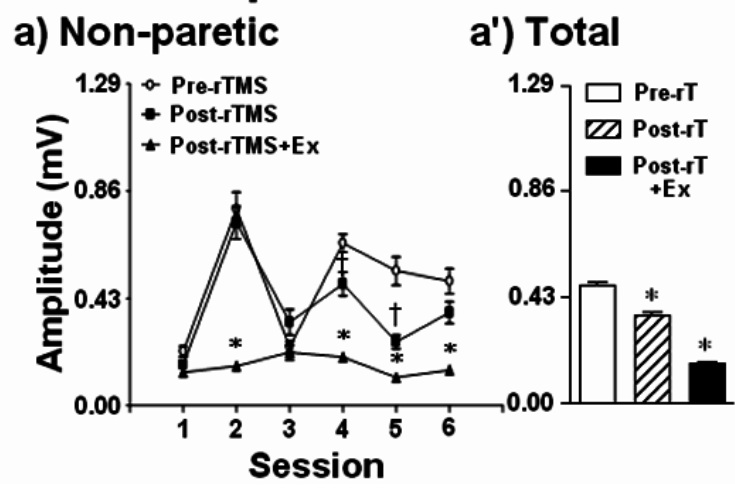

b) Paretic

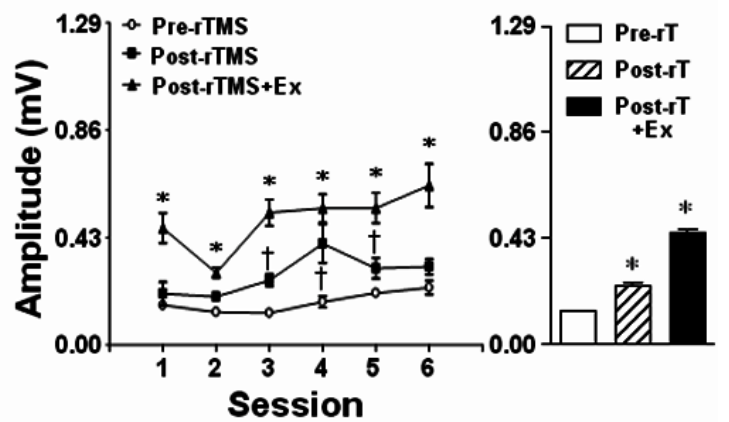

Figure 3. MEP amplitude and latency from both first dorsal interosseous muscles. A and B MEP change over 6 sessions. For each measurement, 25 data points were recorded and analyzed at pre-rTMS, post-rTMS, and post-rTMS + exercise $(n=100)$ $\left({ }^{*}+\mathbf{p}<\mathbf{0 . 0 5}\right)$.

Table 2. Motor function of the upper extremities in stroke patients.

\begin{tabular}{|c|c|c|c|c|c|c|c|c|c|c|c|}
\hline \multirow{2}{*}{\multicolumn{2}{|c|}{ Session }} & \multicolumn{2}{|c|}{ Box and Block (Number) } & \multicolumn{2}{|c|}{ 9-Hole pegboard (Second) } & \multicolumn{2}{|c|}{ Power grip (kg) } & \multicolumn{2}{|c|}{ Pinch grip (kg) } & \multicolumn{2}{|c|}{ Arm reach $(\mathrm{cm})$} \\
\hline & & Pre-rTMS & $\begin{array}{c}\text { Post-rTMS } \\
+ \text { EX }\end{array}$ & Pre-rTMS & $\begin{array}{l}\text { Post-rTMS } \\
\quad+\text { Ex }\end{array}$ & Pre-rTMS & $\begin{array}{l}\text { Post-rTMS } \\
\quad+E x\end{array}$ & Pre-rTMS & $\begin{array}{l}\text { Post-rTMS } \\
+ \text { Ex }\end{array}$ & Pre-rTMS & $\begin{array}{l}\text { Post-rTMS } \\
\quad+E x\end{array}$ \\
\hline \multirow{6}{*}{ 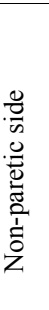 } & $1^{\text {st }}$ & $44.8 \pm 9.4$ & $51.5 \pm 22.5$ & $28.6 \pm 7.5$ & $29.7 \pm 14.0$ & $15.8 \pm 4.8$ & $18.7 \pm 6.1$ & $5.1 \pm 1.2$ & $5.5 \pm 1.0$ & $62.8 \pm 21.1$ & $48.8 \pm 6.5$ \\
\hline & $2^{\text {nd }}$ & $50.5 \pm 25.5$ & $52.0 \pm 23.0$ & $26.5 \pm 11.0$ & $25.1 \pm 9.5$ & $17.0 \pm 5.3$ & $19.2 \pm 5.2$ & $5.4 \pm 0.8$ & $5.6 \pm 1.2$ & $46.3 \pm 9.7$ & $39.5 \pm 1.5$ \\
\hline & $3^{\text {rd }}$ & $54.0 \pm 22.0$ & $51.8 \pm 7.9$ & $26.8 \pm 11.5$ & $25.2 \pm 4.3$ & $18.5 \pm 4.2$ & $17.6 \pm 4.5$ & $5.7 \pm 0.8$ & $5.2 \pm 1.2$ & $45.0 \pm 11.0$ & $73.8 \pm 25.0$ \\
\hline & $4^{\text {th }}$ & $54.5 \pm 21.5$ & $55.5 \pm 19.5$ & $25.5 \pm 9.4$ & $25.0 \pm 8.0$ & $17.8 \pm 4.9$ & $18.3 \pm 4.0$ & $5.8 \pm 0.9$ & $6.1 \pm 0.9$ & $49.2 \pm 23.5$ & $71.7 \pm 41.0$ \\
\hline & $5^{\text {th }}$ & $49.5 \pm 15.5$ & $53.5 \pm 18.5$ & $26.2 \pm 11.0$ & $24.4 \pm 8.9$ & $16.3 \pm 3.0$ & $18.6 \pm 3.1$ & $5.7 \pm 0.8$ & $6.0 \pm 0.7$ & $50.8 \pm 18.5$ & $72.2 \pm 41.2$ \\
\hline & $6^{\text {th }}$ & $56.5 \pm 21.5$ & $51.8 \pm 9.8$ & $26.7 \pm 11.4$ & $24.4 \pm 5.0$ & $17.6 \pm 4.8$ & $17.2 \pm 4.3$ & $5.9 \pm 0.6$ & $5.2 \pm 1.2$ & $50.2 \pm 7.2$ & $68.3 \pm 14.5$ \\
\hline \multirow{8}{*}{ 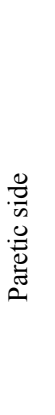 } & Total & $50.8 \pm 5.4$ & $52.4 \pm 4.8$ & $27.0 \pm 3.2$ & $25.4 \pm 2.4$ & $17.0 \pm 1.6$ & $18.1 \pm 1.6$ & $5.5 \pm 0.4$ & $5.5 \pm 1.7$ & $52.4 \pm 6.8$ & $64.5 \pm 9.0$ \\
\hline & $1^{\mathrm{st}}$ & $31.3 \pm 4.3$ & $33.0 \pm 4.0$ & $51.2 \pm 9.8$ & $47.2 \pm 4.4$ & $5.3 \pm 2.1$ & $9.9 \pm 3.0$ & $2.0 \pm 0.8$ & $3.3 \pm 0.9$ & $45.2 \pm 8.9$ & $54.3 \pm 14.7$ \\
\hline & $2^{\text {nd }}$ & $27.5 \pm 6.5$ & $35.0 \pm 5.0$ & $45.6 \pm 4.8$ & $36.6 \pm 0.8$ & $9.2 \pm 4.2$ & $11.0 \pm 3.7$ & $3.3 \pm 1.1$ & $3.2 \pm 0.5$ & $35.7 \pm 1.7$ & $38.5 \pm 0.2$ \\
\hline & $3^{\text {rd }}$ & $33.0 \pm 1.0$ & $37.7 \pm 1.5$ & $45.4 \pm 8.8$ & $34.9 \pm 2.3$ & $11.1 \pm 5.1$ & $6.8 \pm 3.4$ & $3.1 \pm 1.1$ & $2.2 \pm 0.7$ & $34.5 \pm 2.2$ & $44.8 \pm 19.0$ \\
\hline & $4^{\text {th }}$ & $33.5 \pm 1.5$ & $38.0 \pm 2.0$ & $46.7 \pm 3.0$ & $35.4 \pm 0.2^{*}$ & $10.5 \pm 4.9$ & $11.9 \pm 4.6$ & $3.0 \pm 0.9$ & $3.5 \pm 0.9$ & $36.7 \pm 2.3$ & $45.7 \pm 3.7$ \\
\hline & $5^{\text {th }}$ & $34.0 \pm 0.0$ & $37.0 \pm 0.0$ & $40.7 \pm 1.5$ & $37.2 \pm 4.2$ & $10.6 \pm 4.9$ & $12.1 \pm 4.1$ & $3.4 \pm 1.1$ & $3.4 \pm 1.0$ & $43.0 \pm 10.3$ & $54.0 \pm 11.7$ \\
\hline & $6^{\text {th }}$ & $33.0 \pm 1.0$ & $39.3 \pm 2.7$ & $39.8 \pm 0.2$ & $35.4 \pm 2.7$ & $10.4 \pm 5.4$ & $6.8 \pm 3.1$ & $3.7 \pm 1.2$ & $2.8 \pm 0.8$ & $47.7 \pm 6.0$ & $56.3 \pm 8.6$ \\
\hline & Total & $32.0 \pm 1.3$ & $36.9 \pm 1.1$ & $45.4 \pm 2.5$ & $37.4 \pm 1.4^{*}$ & $8.9 \pm 1.4$ & $9.0 \pm 1.4$ & $2.9 \pm 0.4$ & $2.9 \pm 0.3$ & $41.1 \pm 3.0$ & $49.2 \pm 4.6$ \\
\hline
\end{tabular}

${ }^{*} \mathrm{p}<0.05$. 
Table 3. Correlation between motor function and MEP.

\begin{tabular}{ccccc}
\hline & \multicolumn{2}{c}{ Paretic side } & Non-paretic side \\
\hline & Amplitude & Latency & Amplitude & Latency \\
\hline Box and Block & 0.29 & $-0.806^{* *}(0.000)$ & 0.255 & -0.284 \\
9-Hole pegboard & $-0.486^{*}(0.01)$ & -0.12 & -0.325 & 0.328 \\
Power grip & -0.024 & $-0.423^{*}(0.02)$ & 0.351 & -0.02 \\
Pinch grip & -0.046 & $-0.569^{* *}(0.001)$ & $0.422^{*}(0.02)$ & 0.059 \\
Arm reach & $-0.371^{*}(0.043)$ & $-0.7^{* *}(0.000)$ & 0.214 & -0.24 \\
\hline $\mathrm{p}<0.05 ;{ }^{* *} \mathrm{p}<0.01$ & & & &
\end{tabular}

has combined physical therapy with rTMS; nevertheless, we can still compare our results with other studies that adapted motor training with rTMS $[15,18,19]$. These studies also reported increased effects of rTMS when it accompanied motor training. We found consistent results of motor function improvement between post-rTMS and post-rTMS + exercise in the paretic upper extremity in the 9-hole pegboard test during the overall procedure, whereas the non-paretic upper extremity showed no continuous changes. The results cannot be overlooked, especially since continuous improvement was seen for the 9-hole pegboard task, the most dexterity-demanding task among our evaluation tools, in the paretic upper extremity. This may be due to our unique design of ROM exercise intervention that is guided appropriately by a physical therapist. Previous studies combining motor function with rTMS conducted repetitions of simple movements $[15,19]$. The upper extremity movement is complex and needs coordinated activation of several different muscles [20]. We also saw trends toward functional improvement between the 1st and final sessions at pre-rTMS in the paretic hand in every motor function test. Motor improvement in the paretic upper extremity corresponded with increases in MEP amplitude on the ipsilesional M1 that were augmented as sessions progressed. This suggests that motor function improvement in the paretic upper extremity is involved with MEP changes in the ipsilesional hemisphere. This result is in accordance with previous studies that reported that MEP amplitude is related to motor recovery [21-23]. Kim et al. who investigated the relationship between MEP amplitude and motor function, found a significant correlation between MEP amplitude and motor accuracy [19]. Takeuchi et al. indicated that both force of pinch grip and MEP amplitude were increased after application of rTMS and motor training [15]. The results of the present study suggest significant correlations between MEP and motor function. The Box and Block, force of pinch grip, and arm reach test results were strongly correlated with MEP latency and the 9-hole pegboard and arm reach test results were correlated with MEP amplitude in the paretic upper ex- tremity. The force of pinch grip was also correlated with MEP amplitude in the non-paretic upper extremity. Therefore, our motor evaluation tool can be considered appropriate for measurement of motor recovery following changes in cortical excitability. The present study has some advantages. First, it is the first to adopt physical therapy combined with rTMS for stroke patients. As previous studies reported, additional physical training may accentuate the effect of rTMS and lead to functional recovery after stroke $[15,18]$. Thus, ROM exercise guided by experienced physical therapy after application of rTMS is an appropriate therapy in this respect. In the present study, ROM exercise after rTMS resulted in a greater excitation of the ipsilesional M1 than when it was performed immediately after rTMS. This implies that ROM exercise can augment the effect of rTMS and can contribute to motor recovery. Although no previous study has applied physical therapy with rTMS, some studies have conducted simple motor training with rTMS $[15,19]$. According to the authors, motor functions were improved after motor training as well as after application of rTMS. The ROM exercise and evaluation tools used in this study were designed by considering both actual complex upper extremity movements such as reach and grasp [2] and overlapping brain mapping in the motor cortex [20]. Therefore, we performed ROM exercises from the elbow to the finger joints and used the Box and Block and 9-hole pegboard tests. Correlation analysis in this study also supports the relevance of our motor evaluation tool to detect changes in motor excitability. In a similar respect, Mansur et al. studied the effect of rTMS on upper extremity function as measured by the Purdue pegboard task [24]. Participants showed an improved performance after $1 \mathrm{~Hz}$ rTMS in the pegboard task. However, these researchers only measured the pegboard task without additional motor training, unlike the present experimental design. A previous study conducted using 5 days of rTMS reported a cumulative effect that lasted for 2 weeks [14]. We can expect more powerful and long lasting effects from our study protocol because the procedure consisted of one more session ( 6 days for 6 ses- 
sions) and we added physical therapy after rTMS to every single session. This study has some limitations that should be examined. First, the quite long duration (1.5 hrs) of each session due to the additional physical therapy after rTMS might have given rise to fatigue, which could have interfered with our results. Second, some participants were excluded during measurement of TMS parameters due to weak responses based on our standard. Therefore, we collected 25 responses in every MEP evaluation (pre-rTMS, post-rTMS, post-rTMS + exercise) to obtaining the mean MEP measurements. In summary, cortical excitability in both hemispheres was continuously changed both after rTMS and after ROM exercise and it was greatly increased after exercise. Motor evaluation tests showed a correlation with MEP. Physical therapy combined with rTMS is suggested as a protocol to facilitate motor recovery of stroke patients.

\section{REFERENCES}

[1] N. Takeuchi, T. Tada, M. Toshima, T. Chuma, Y. Matsuo and K. Ikoma, "Inhibition of the Unaffected Motor Cortex by $1 \mathrm{~Hz}$ Repetitive Transcranical Magnetic Stimulation Enhances Motor Performance and Training Effect of the Paretic Hand in Patients with Chronic Stroke," Journal of Rehabilitation Medicine, Vol. 40, No. 4, 2008, pp. 298303. doi:10.2340/16501977-0181

[2] A. Peinemann, B. Reimer, C. Loer, A. Quartarone, A. Munchau, B. Conrad and H. R. Siebner, "Long-Lasting Increase in Corticospinal Excitability after 1800 Pulses of Subthreshold $5 \mathrm{~Hz}$ Repetitive TMS to the Primary Motor Cortex," Clinical Neurophysiology, Vol. 115, No. 7, 2004, pp. 1519-1526. doi:10.1016/j.clinph.2004.02.005

[3] A. J. Butler and S. L. Wolf, "Putting the Brain on the Map: Use of Transcranial Magnetic Stimulation to Assess and Induce Cortical Plasticity of Upper-Extremity Movement," Physical Therapy, Vol. 87, No. 6, 2007, pp. 719736. doi:10.2522/ptj.20060274

[4] H. M. Feys, W. J. de Weerdt, B. E. Selz, G. A. Cox Steck, R. Spichiger, L. E. Vereeck, K. D. Putman and G. A. van Hoydonck, "Effect of a Therapeutic Intervention for the Hemiplegic Upper Limb in the Acute Phase after Stroke: A Single Blind, Randomized, Controlled Multicenter Trial," Stroke, Vol. 29, No. 4, 1998, pp. 785-792. doi:10.1161/01.STR.29.4.785

[5] A. Sunderland, D. J. Tinson, E. L. Bradley, D. Fletcher, R. Langton Hewer and D. T. Wade, "Enhanced Physical Therapy Improves Recovery of Arm Function after Stroke. A Randomised Controlled Trial," Journal of Neurology, Neurosurgery \& Psychiatry, Vol. 55, No. 7, 1992, pp. 530535. doi:10.1136/jnnp.55.7.530

[6] C. N. Tseng, C. C. Chen, S. C. Wu and L. C. Lin, "Effects of a Range of Motion Exercise Programme," Journal of Advanced Nursing, Vol. 57, No. 2, 2007, pp. 181191. doi:10.1111/j.1365-2648.2006.04078.x

[7] C. Hofgren, A. Bjorkdahl, E. Esbjornsson and K. Sti-
brant-Sunnerhagen, "Recovery after Stroke: Cognition, ADL Function and Return to Work," Acta Neurologica Scandinavica, Vol. 115, No. 2, 2007, pp. 73-80. doi:10.1111/j.1600-0404.2006.00768.x

[8] Y. H. Kim, S. H. You, M. H. Ko, J. W. Park, K. H. Lee, S. H. Jang, W. K. Yoo and M. Hallett, "Repetitive Transcranial Magnetic Stimulation-Induced Corticomotor Excitability and Associated Motor Skill Acquisition in Chronic Stroke," Stroke, Vol. 37, No. 6, 2006, pp. 1471-1476. doi:10.1161/01.STR.0000221233.55497.51

[9] N. S. Ward and L. G. Cohen, "Mechanisms Underlying Recovery of Motor Function after Stroke," Archives of Neurology, Vol. 61, No. 12, 2004, pp. 1844-1848. doi:10.1001/archneur.61.12.1844

[10] C. Patten, J. Lexell and H. E. Brown, "Weakness and Strength Training in Persons with Poststroke Hemiplegia: Rationale, Method, and Efficacy," Journal of Rehabilitation Research and Development, Vol. 41, No. 3A, 2004, pp. 293-312. doi:10.1682/JRRD.2004.03.0293

[11] J. V. Escudero, J. Sancho, D. Bautista, M. Escudero and J. Lopez-Trigo, "Prognostic Value of Motor Evoked Potential Obtained by Transcranial Magnetic Brain Stimulation in Motor Function Recovery in Patients with Acute Ischemic Stroke," Stroke, Vol. 29, No. 9, 1998, pp. 18541859. doi:10.1161/01.STR.29.9.1854

[12] F. Fregni, P. S. Boggio, A. C. Valle, R. R. Rocha, J. Duarte, M. J. Ferreira, T. Wagner, S. Fecteau, S. P. Rigonatti, M. Riberto, S. D. Freedman and A. Pascual-Leone, "A Sham-Controlled Trial of a 5-Day Course of Repetitive Transcranial Magnetic Stimulation of the Unaffected Hemisphere in Stroke Patients," Stroke, Vol. 37, No. 8, 2006, pp. 2115-2122. doi:10.1161/01.STR.0000231390.58967.6b

[13] C. G. Mansur, F. Fregni, P. S. Boggio, M. Riberto, J. Gallucci-Neto, C. M. Santos, T. Wagner, S. P. Rigonatti, M. A. Marcolin and A. Pascual-Leone, "A Sham Stimulation-Controlled Trial of rTMS of the Unaffected Hemisphere in Stroke Patients," Neurology, Vol. 64, No. 10, 2005, pp. 1802-1804. doi:10.1212/01.WNL.0000161839.38079.92

[14] N. B. Lincoln, R. H. Parry and C. D. Vass, "Randomized, Controlled Trial to Evaluate Increased Intensity of Physiotherapy Treatment of Arm Function after Stroke," Stroke, Vol. 30, No. 3, 1999, pp. 573-579. doi:10.1161/01.STR.30.3.573

[15] B. J. Brouwer and K. Schryburt-Brown, "Hand Function and Motor Cortical Output Poststroke: Are They Related?" Archives of Physical Medicine and Rehabilitation, Vol. 87, No. 5, 2006, pp. 627-634. doi:10.1016/j.apmr.2006.02.006

[16] N.Takeuchi, T. Tada, T. Chuma, Y. Matsuo, I. Watanabe and K. Ikoma, "Repetitive Transcranical Magnetic Stimulation of Contralesional Primary Motor Cortex Improves Hand Function after Stroke," Stroke, Vol. 36, No. 12, 2005, pp. 2681-2686. doi:10.1161/01.STR.0000189658.51972.34

[17] S. Pak and C. Patten, "Strengthening to Promote Functional Recovery Poststroke: An Evidence-Based Review," 
Topics in Stroke Rehabilitation, Vol. 15, No. 3, 2008, pp. 177-199. doi:10.1310/tsr1503-177

[18] I. Indovina and J. N. Sanes," On Somatotopic Representation Centers for Finger Movements in Human Primary Motor Cortex and Supplementary Motor Area," Neuroimage, Vol. 13, No. 6, 2001, pp. 1027-1034. doi:10.1006/nimg.2001.0776

[19] M. A. Diego, T. Field, M. Hernandez-Reif, S. Hart, B. Brucker, T. Field and I. Burman, "Spinal Cord Patients Benefit from Massage Therapy," International Journal of Neuroscience, Vol. 112, No. 2, 2002, pp. 133-142. doi:10.1080/00207450212023

[20] P. E. Morris, "Moving Our Critically Ill Patients: Mobility Barriers and Benefits," Critical Care Clinics, Vol. 23, No. 1, 2007, pp. 1-20. doi:10.1016/j.ccc.2006.11.003

[21] N. J. O'Dwyer, L. Ada and P. D. Neilson, "Spasticity and Muscle Contracture Following Stroke,” Brain, Vol. 119,
Pt. 5, 1996, pp. 1737-1749. doi:10.1093/brain/119.5.1737

[22] H. T. Hendricks, J. van Limbeek, A. C. Geurts and M. J. Zwarts, "Motor Recovery after Stroke: A Systematic Review of the Literature," Archives of Physical Medicine and Rehabilitation, Vol. 83, No. 11, 2002, pp. 1629-1637. doi:10.1053/apmr.2002.35473

[23] A. A. van Kuijk, J. W. Pasman, H. T. Hendricks, M. J. Zwarts and A. C. Geurts, "Predicting Hand Motor Recovery in Severe Stroke: The Role of Motor Evoked Potentials in Relation to Early Assessment," Neurorehabilitation and Neural Repair, Vol. 23, No. 1, 2009, pp. 45-51. doi: $10.1177 / 1545968308317578$

[24] N. Murase, J. Duque, R. Mazzocchio and L. G. Cohen, "Influence of Interhemispheric Interactions on Motor Function in Chronic Stroke," Annals of Neurology, Vol. 55, No. 3, 2004, pp. 400-409. doi:10.1002/ana.10848 